\title{
Bayesian Analysis of the Sudanese Immigrant Youth Crime Rates and the "Likelihood" of Committing Violent Offence than an Australian-Born
}

\author{
Atemthii D. Dau* \\ Sudanic Centre For YouthLinks and Community Social Wellbeing, South Sudanese Community Centre, \\ Footscray, Australia
}

\begin{abstract}
This paper presents a mixed method of inquiry into most of the public notions, shaping the Sudanese immigrant community's perception Australia wide. Firstly, a qualitative review regarding two remarks by Australian public figures will be considered and analyzed; and secondly, Bayesian analysis (BA) will be considered to analyze the randomness of the crimes: BA, is a highly predictive methodological tool used in a wide range of applications. For example, in predicting of crimes based on prior occurrences of an offence or groups of offences. Thus, the Bayesian analysis considers the hypothesized relationship between 'Ethnicity and Criminality'; the emphasis is on the recorded Crime figures involving immigrant youth of the Sudanese-born residing in the state of Victoria. The figures are drawn mainly from the Australian statistical agencies and media sources; the Australian Bureau of Statistics (ABS), media reports, community's prison population (CPP) and the overall Sudanese immigrant population in Australia (SIPA); comparative considerations with the overall Australian population (APP) and the Australian-born prison population (ABPP) from the years 2006 to 2007 will be looked into. The study concludes by suggesting the policy implication of this findings and future research directions.
\end{abstract}

Keywords: Bayesian analysis, Ethnicity, Criminality, Sudanese immigrant youth, Crime rates.

\section{INTRODUCTION}

The aim of this study is to analyze the effects of conflictive intercultural reactions on participation of individual member of Sudanese immigrant group in state or neighborhood's crime rates and the likelihood of committing or conviction of criminal offence than an Australian-born individual and other ethnic minority group in Australia. Dan Oakes (2012) hypothesized on a relationship between Sudanese Immigrant youth and the statistical level of crimes in Victoria; concluding that ethnic youth, and in particular, the Sudanese-born (SB) are almost up to five times more likely to commit a violent offense than their Australian-born (AB) counterparts (Oakes 2011); accordingly, they are responsible for heightening the state's crime records.

Hence, following Oakes' hypothetical conclusion triggered an unprecedented, yet conflicting public response, chiefly through the media. Oakes findings conflict with the then Victorian Police Commissioner and the Victorian Police Union. Besides public panic that it caused, media portrayal of the Sudanese Immigrants continues to present them as perpetrators of crimes and therefore a threat to the Australian way of life (Nolan et al. 2011). Thus, the link between Sudanese refugees and criminality in Australia seems

*Address correspondence to this author at the Sudanic Centre For YouthLinks and Community Social Wellbeing, South Sudanese Community Centre, Footscray, Australia; Tel: +61412545997; E-mail: atemddau@gmail.com confirmative to two key events: (1) an earlier inflammatory remark by Andrew Fraser on the Sudanese Immigrants, regarding race and intelligence (IQ); and (2) the commonwealth government official response that terminated or frozen Sudanese immigration into the country, claiming total lack of integration by the community into Australian mainstream. The empirical results provide important insights on the determinants of criminal offences. The findings reveal that the reported crime rate perpetuated by the Sudanese immigrant youth is a lot higher than their overall prison population; equaling the total Sudanese Immigrant population (SIP), Australia wide. In that, Oakes' conclusion, however, controversial it seems, is has be shown to be unattainable.

There appears to be no significant relationship between: either the rate of participations in crime among immigrant youth, suggesting the nature of the offence, or the cultural or ethnic background, as well as the age of the convicted individuals, that could act as intervening variables are unsupported by the results in this analysis. Therefore, the main hypothesis is that Sudanese immigrant youth has a lower participation in crime events; they are less likely to commit a crime or convicted of homicide crime than those of Australianborn in Melbourne, Australia. This hypothesis is framed in Bayesian techniques: a probabilistic likelihood theory; and content analysis for public discourse, specifically media representation of Sudanese immigrant populations in Australian crime statistics. 
Theoretically, the link between individual committing a crime (criminality) and his or he ethnic (race) or cultural background is ambiguous. For instance, Andrew Fraser (2005), put forth the notion of "race" and criminality", which is rooted in the concept of "RacePolicy" fallacy. The idea is that individuals of the new arriving 'black' Africans-race to Australia, and in particular a Sudanese immigrant persons, are characterized as nothing less than a population of criminal 'gangs', and negative perception such as violence, theft and assault attached to them; and thus, they are perceived to have substantial contribution in states' or neighborhoods' crime rates. This results in an imprisonment (punishment), or likelihood of committing an offence being negatively associated with an individual's appearance in this group. In this scenario, not only are imprisonments not a race-ethnicity, but also encouraging individuals to commit more crimes, insofar as they are labeled as 'criminals' or 'gangs'. Certainly, it is not a case for this group, although, in some instances, punishment (imprisonment) has been found and perceived to be associated with lower risk of participations in crime (Anwar 2011; Tonry 1994).

\section{MEDIA, POLITIC AND RACE-CRIMINALITY}

The first media attention on the Sudanese immigrants in Australia was spearheaded by the then Macquarie university professor of law, Andrew Fraser in the year 2005, as mentioned earlier on; Fraser characterizes the community as nothing less than a population of violent 'gangs', taking refuge in Australia. Fraser calls for a commonwealth government's total cancelation of the humanitarian visas for the Sudanese refugees immigrating into Australia; thereby linking race or ethnicity with criminality. Although the professor's remarks were publicly condemned for racial slurs and a complete lack of empirical evidence, though he refers to the United States' (U.S) crimes data regarding African-Americans or 'blacks' prison population and the crimes they commit individually; but believes being 'black', implies an individual's lower level of intelligent Quotient (IQ), seeing it as mediating or contributing factor to their participations in states' or neighborhood's crime rates, and also, as the basis for disorderly behavior-or- be prone to crime; disrupting or breaking the well-established socio-legal systems: best practices of law and order by enforcement agencies-orauthorities across administrative states and territories. Thus, the basic feature, which Fraser developed against the Africans, or black Sudanese, hypothesizing the relationship between race and criminality, received its due credibility in a form of response by some public politicians, marking the second phase for the Sudanese media's scrutiny, and what seems to be the confirmation of the Frasers hypothesis. When the then immigration minister, Kevin Andrews announced his Department's 'official' policy position on the Sudanese immigration into Australia terminating the arrival of 'who would-be Australians' by joining their family members (Cooks 2007;DIAC 2009), as part of the sub-Saharan African immigrant communities in Australian community (Nolan et al. 2011); Australia-a country, which prides itself as true immigrants nation.

There are legitimate reasons of assuming the agreement between the Andrew and Fraser, for it is not so much an attitude towards immigration itself, but the two's views as to what Sudanese criminal behavioror-black mentality that Fraser raises, gauged by the media and implemented by the then Immigration Minister, Kelvin Andrew (Nolan et al. 2011). Failure to successfully integrate is true 'un-Australia', but whether Kelvin Andrew is entirely free from Fraser's contention that, black does mean an individual's predisposition to Crimes, irrespective of place. At the face of this 'Racepolicy' issue, it is fair to say that minister Andrews did not publicly cite 'race' as warranting his total freeze of the humanitarian applications for the Sudanese refugees. Herein, Andrews remains unapologetic about his stance on African refugees, rejecting accusations he has fuelled violence involving Sudanese migrants and having living difficulties or other social problems integrating to Australian community (Davidson 2011; Nolan et al. 2011).

\section{The Qualitative Approach Arresting the Immigrants by their Intelligence Quotient (IQ): Andrews and Fraser on Race, Crimes, and Immigration}

A closer look into the minister's excerpts brings him in agreement with that Fraser's contention of black's $I Q$, as black's behavior. Although, the community has been victimized-by government officials, from the highest levels of the Australian; Kelvin Andrews, like Fraser before him remains unapologetic about his remarks and the damage it has caused the community. For example, a Sudanese-born teenager was attacked by four [white] men in suburb of Melton in Victoria, Australia, just a day after Minister's policy announcement. The Australian-born assailants stole [victim's] wallet, and mobile phone, and then sent racist text messages and phone calls to his brother. Therefore, if we examine Andrews' claim of the immigrant community's total failure in its 'integration' process, as warranting his total freeze of all the 
humanitarian applications into Australia by the Sudanese refugees. And although, Andrew's departmental response did not also mention 'criminality', it does amount to the uniformity, Or universality of a black population, or-black's behavior, which is Frasers exact position and hence their agreement. In the same way that minister Andrew did not utter the word, race; albeit, indirectly and argues his case entirely on different platform-but the same. Fraser was the first, in hypothesizing the relationship between black, and in particular, sub-Saharan Africans and their IQ level, as limited to racial pigmentation. In this sense, however, it seems that Sudanese immigrants-individuals,' or other sub-Saharan African's physical appearance implies a behavior that is punishable by laws or criminalized outright.

It is used in criminological explications, involving criminals' past, or previous offences and the likelihood or the probability of doing the same offences. Myers et al. (1990) defines Bayesian probabilistic analysis as, partition of events or the sample Spaces, $\mathbf{S}$ into 'subsets', with the prior occurrences of events not be same or equal. In considering the relationship between Sudanese immigrant youth's crime rates participation and the overall state's crimes record in the state of Victoria. Bayesian analysis does reveal that, the findings on which the Australian public discourse heavily relies on is unsubstantiated. The suggestion, according to Oakes (2012) findings, a Sudanese immigrant youth is up to five times more likely to commits violent crimes than his or her Australian-born counterpart is not based on empirical evidence. Here, 'violent crime' refers to: homicide, sexual or physical assault or material extortion or robberies. However, as the recorded data shows, and in the words of Sinclair Davidson (2011) that, "criminal behavior is related to opportunity cost and that would be weakly related to ethnicity, if at all, and given the fact that Australia as a liberal society does not concern itself with the ethnic criminals" (Davidson 2011, p.4). But, even-though I agree with Davidsons' main assertion and assessment, Australia does seem have: 'ethnic criminals', or gangs; base on individuals' ethnic background or native country of birth.

\section{Immigration, Ethnicity, and crime}

Previous researches on issues of immigration and ethnicity have raised three conventional contentions. The first contention is that: immigrants often encounter problems of acculturation and assimilation [integration] At entering and during the process of adjustment to new host community-Australian community (Agher \& Strange 2008). This is based on the belief that an immigrant seems to strike a balance-complex negotiation between two cultures; (1) immigrants' native cultures; and (2) cultures of the receiving community (dominant mainstream group), which later shrinks, becoming a non-dominant and often at verge of or in-fear of extinction: vanishing of ones' own native cultures (Grace and Marta 1995; Mesch et al. 2008), only, often in favor of new cultures of the new receiving community. Which gradually, comes as a result of resettlements and integration process, most of which, cultures of the receiving community, considerably, gain its acceptances and become dominant over immigrant ethnic cultures; replacing an immigrants' own cultures. This process is generally termed as the acculturative process. Language or communication generalizes this process (Collins 2005; Grace \& Marta 1995; Tonry 1994). The second is conflict-laden contention and considers an ethnic group versus another and shows that a number of immigrants from different ethnic minorities may commit crime at "disproportional" rates compared to other groups (Collins 2005; Tonry 1994). This suggests that the immigrant crime participation rates may be either higher or lower, but not both. Furthermore, the final theoretical contention considers the demography of the immigrants as a whole and divides researchers into those who agree and those who do not. The middle, however, is that they both argue that, immigrant groups differ from others (host, or ethnic minorities) on: age and gender composition, socio-economic status, wealth and other social status, which strongly characterizes a higher level of deviance behavior, as indicative of criminality among most socially and economically disadvantaged immigrant communities in their countries, especially, wellestablished or developed nations, like Australia (Gifford et al. 2007; Tonry 1994). This suggests that, conflicts between different ethnic immigrant groups and that hosting society, perhaps, may significantly contribute in crimes rates in hosting societies, for example, Australia hosting Sudanese immigrants or refugees, notwithstanding. The conflicts are mainly due to an immigrant economic conditions, which varies from one immigrant to another (immigrant), during his or her acculturative process. This seems, however, that before both Andrew and Fraser public inflammations, which centralized the community as gangs, in around Australian's major cities, but there was no reports of property or any valuable material thefts by the young Sudanese immigrants did occur. But, what did occur and presumably as the youth's own response to state's 
police targeting them was when a detective was punched and kicked after confronting about 20 Sudanese young men in the Melbourne suburb of Noble Park, a reputed trouble spot of gangs (Cooks 2007; Theage Newspaper 2007). Thus, we observe that, the stolen material belongs to a Sudanese-born immigrant and not to his Australian-born counterparts; hence, the Noble Park youth were by no means a 'gangsters'. This suggests that in the above two incidences-both economic, or 'gangster-ism'-that is; it is the Sudanese-born persons rather than their counterparts: the constable deductive-or-the Melton assailants, which belongs to the assault victim and the Australian-born, clearly, the 'assailants' and against the member of immigrant ethnic groups, the Sudanese immigrant community.

However, given the contingencies regarding the crimes, it does appear that although the economic differences might shape the social behavior of immigrants' settlement and their interaction with the wider host society, or facing the host nation's criminal system; other theories disagree with the economic conditions of an immigrant as responsible entirely for the behavior.

Instead, Tonry (1994) these behavioral theorists contend that immigrants may psychologically be predisposed to some confronting positions-such as the youth's encounter with the deductive; and as such, immigrants do face psychological conditions, however, varied it may be. The 'variety', here, might itself mean behavior; and it is what determines what a host society might view as amounting to criminal behavior itself. However, behavior does not necessarily mean 'criminal'. As, Tonry (1994) observes, behavioral differences, for example, between different ethnic immigrant groups; and within a single host societycommunity, do not reflect much on the "arrests and imprisonment disparities". Tonry believes that, behavior is a result of social conditions, including; the inherited poverty, socio-economic status, or lack of economic opportunities that are encountered by immigrants, for example: the Sudanese immigrants at entering into Australia. Therefore, it's the social or the acculturative process of adapting into a host society, rather than race: skin color, as Fraser contends - and possibly Oakes' position that, would lead immigrant groups to commit more criminals than their native-born counterparts, insofar as those, arrests and imprisonments are primarily due to their behaving different, rather than being correctly charged for due offences. The arrests and imprisonment, if at all recorded against a Sudanese Immigrant, is regarded as a data and will be considered in that form rather than in their own right. As this, the above theoretical position revealed that, Frasers' contention to be perceived, as faintest argument and the most racially predisposed argument against or in relations to Sudanese immigrant populations in Australia. Hence, it appears that, Oakes' conclusion about Sudanese immigrant youth and crime rates, relies and is anchored on two contradictory positions, on the one hand, and their skin-color, cultural and ethno-racial backgrounds, as measure variables or valuable indicator to determine their participation or involvements in criminal activities, such as: violent crimes and other types of physical aggression, on the other.

It therefore, lacks the predictive powers that empirical data should have, in order to examine the basic features of the study variables and how they are related to the dependent variables. In that, Oakes rely on another ambiguous consideration: the Sudaneseborn (SB) individual, which is not the same, as the Sudanese-descent (Sudanese-by-ancestry) person in the community. Ethiopians, Somalis, and Eritreans, who have first, took residence in the country, Sudan, as refugees do, in fact have the Sudanese-born of their own, in their respective communities. According to Australian Bureau of Statistics (ABS), Sudanese immigrant populations in Australia, is categorized into two group:(1) firstly, is the Sudanese-by-birth (SB); and (2) secondly, is the Sudanese-by descent (SD); while, the two sub-Sudanese immigrant populations does not makes up, the overall Sudanese immigrant population in Australia (SIPPA). It does also; suggest that, the Sudanese-born (SB), which Oakes considers cannot be a true representative of the criminal records of the Sudanese Immigrant Community or of its youth. This therefore, is being a standardization of the total Sudanese immigrants populations in Australia (SIPPA). The Sudanese-by-birth (SB) and the Sudanese-by ancestry (descent) (SD), are distinct from the each other; hence Oakes' conclusion is shown to be an ambiguous position and therefore, unattainable.

\section{SUDANESE IMMIGRANTS IN AUSTRALIA}

Sudanese immigration into Australia began in the earlier 90s and dwindles in the mid-2000s. As of 2006 census, there were 19,369 Sudanese-born in Australia, compared to 4,910 in 2001 (ABS 2005), making up $0.01 \%$ of the [Australian] population. This means that, in a random population of a thousand people, one is a 
Sudanese. The population is of two groups: the Sudanese-by-birth and Sudanese-by-ancestry, which makes total population of the Sudanese immigrant in Australia; however, there is a significances difference between Sudanese-by-birth and Sudanese-byancestry. Such key differences remain, somewhat blurred to Australian statistical agencies, when reporting crimes, in one sense, and in particular in Melbourne, where once again, 'they' make-up to, 6,085 of the Victorians' population (ABS 2011).

However, it remains unclear as to whether the African-Victorians or Australians' population is either predominantly: a Sudanese-by-ancestry or a Sudanese-by-birth. But, it is plausible to assume that the Sudanese immigrant population in Australia, concerning either of the two, is composed of the Sudanese-by-birth and the Sudanese-by-ancestry. Of the two, however, the Sudanese-by-ancestry makes up 17,186 of Sudanese migrants in Australia (Australian Bureau of Statistic 2006) ${ }^{1}$, whereas the Sudaneseborn persons recorded in the 2006 Australian census had arrived very recently, making a total of 19,369 Sudanese persons among Australian residents (ABS 2010), most of which, $77 \%$ of this population arrived to Australia between 2000 and 2006 (ABS 2006; DIAC 2009). And from, 2002 to 2007, the intakes of refugees from Sudan peaked, increasing by $28 \%$ per year; eventhough the community remains one of the ethnic minority groups in comparison to the Australian overall population.

\section{But, if all Crimes are Crimes: How much is Ethnical?}

In much broader contexts, literature and theories of Immigration do also consider, immigration, Ethnicity and Crime, as significant factors shaping an immigrant re-settlement into the new hosting society (Krieger et al. 2005; Thomas 2011). Therefore, Fraser and Oakes claims will be examined in lights of the existing theories linking, ethnicity and crimes in Australian context (Thomas 2011); and the focus, is on the links between Sudanese immigrants and crime rates in the state of Victoria, Australia.

If, according to various claims, the Sudanese immigrant youth are, approximately, about five (5) times more likely to commit and to be persecuted for a homicide than the Australian-born (AB) individuals; and given the 'ethnical', or perhaps, 'natural-or-cultural differences', with the predominately white and crimefree Australian. As Fraser contends, the Sudanese
Immigrant youth are also up to eight (8) times more likely to violently and naturally assault their victims than the Australian-born; and are three (3) times more likely to sexually assault and more than three times to be convicted for material 'extortion or robbery' than the Australian-born (AB) individuals (Cooks 2007; Fraser 2005; Oakes 2012). If such a statistical data were correct, it should have revealed the Sudanese Immigrants as the biggest criminal network throughout Australia and could possibly be found in Australian prisons, serving their respective: homicides, violent or sexual assaults, and robberies or extortions jail terms. And if Fraser is correct on his race and lower levels of intelligence (IQ) entailing criminality as per nature of blacks, the entire SIPPA could possibly be serving its jail terms or has just been out. The latter, however, represents 'certainty' and not the 'likelihood' that this paper concerns itself with.

However, theoretical and previous studies on immigration and crimes, have found no significant relationship between ethnicity and crimes, or delinquency. For example, Xi Chen and Hua Zhong (2013) believe that classical theories on immigrationor crime studies have always focused on culture and contend that immigrant youth are less delinquent than native-born adolescents (Montgomery \& Foldspang 2008). This suggests that, empirical studies on immigration, ethnicity, and crime do conclude that, there is no sufficient evidence; that is to say, there isn't an established relationship or the correlation between the population level of ethnic immigrants and the crimes that, they perpetuate. In brief, the higher the number of ethnic immigrant in a country, does not necessarily lead to higher crimes rates (O'Sullivain \& Olliff 2006; Tran 2005; Lee \& Martinez 2001).

\section{Sudanese Prison population in Australia (SIPPA)}

Oakes, who may be regarded, as the key contender for his supplies of the statistical, or the probabilistic likelihood of the Sudanese immigrant youth; supplanting both Fraser the minister's, maintains that as, of the 2010. The total prison in-mates countrywide stood at 29, 300 convicted individuals. Of this, 77 prisoners were of Sudanese- born (SB), convicted of homicide or related offences; and 1,682 Australianborn individuals convicted of the same offences (Oakes 2012). Oakes clearly stated that Sudanese-born immigrant youth in Victoria, particularly in Melbourne, are responsible for heightening the rates of the states' crimes, and concludes that the Sudanese immigrant youth, are almost up-to five times more likely to commit 
a violent offence than their Australian-born counterpart, presumably, of the same age group or the cohorts (Oakes 2012). However, the two contrasting positions, remarkably, stand out to Oakes contentions. The first regard is, the Victorian police crime figures, and in particular the institution's former commissioner, Christine Nixon's view(s), which puts her at odds with the institutions own union, regarding, the involvements of the Sudanese immigrant youth in violent crimes or other criminal offences and their contribution to the states' or neighborhoods' crime rates. The then, Victorian Police commissioner, Christine Nixon in her interview with 3 WA radio, had this to say: "when you look at the data that l've looked at, it does seem to me that there is a higher proportion" (Cooks 2011; Heraldsun Newspaper 2010).

On the other hand, Davidson (2011), in an editorial article over the participations of immigrant persons in state crimes, referred to the following title: 'Who is Foreign Criminals?' disagrees with Oakes' conclusion. For Davidson, Oakes has not presented empirical evidence to support the claims that, the Sudanese immigrant youth, are almost up-to five times more likely to commit or to be convicted of violent crimes including homicide events than their Australian-born counterparts, presumably, of the same age group. In Davidsons' view:

The crime rates per 100,000 of population relative to the crime rate for Australianborn non-indigenous per 100,000 looks very high for some groups - but there is a perception bias in reporting the stats like that. It is not reasonable to believe that any foreign born group would have no individuals in prison for any crime and the smaller that group the higher the population weight is going to be everything else being equal (Davidson 2011,p.2).

\section{BAYESIAN MODEL AND ANALYSIS}

In what will thus follow, I proceed to firstly, present the intended Bayesian analysis; and secondly the discussion(s) that, is based on the ABS data periods from 2006 to 2007; and 2010 to 2012 and, those supplied by Oakes, in his 2007-2012 reports on Victorians crime rates, titled as: African youth Crime Concern (2012); Fear of cronulla -like unlike as refugee lawlessness grows in Melbourne (2012) reportages. However, as I made it clear in both, the title and discussion herein, that my main focus, is on the
Sudanese immigrant youth in victoria-Melbourne. I maintain that neither Oakes' nor the ABS figures warrant the assertion, which heightens the Australians public discourse and the beginning of racial assaults, racist-behavior on, and negative perceptions of Sudanese Immigrant groups; apparently in the media discourse: relying on figures in Oakes' reports, the ABS figures and Sudanese Immigrant youth's total prison population. In the latter case, however, Oakes maintains, as of 2009 Australian prison population, which stood at 29, 300 prisoners convicted of similar offences: this include, the Sudanese-born ( 77 out of 29,300 prisoners), and Australian born (1,682 out of 29,300 prisoners). This, however, is disputable, Sudanese immigrant community is constitutive of two different groups: the Sudanese-by-birth and Sudaneseby-ancestry, according to ABS (2006). The community is distinctively, Sudanese-by-ancestry. Sudan, as country, previously, hosted refugees from Ethiopia, Eritrea, and the Somalia, before settling into Australia; and whose young members of the respective communities are classified, as Sudanese-born; and hence, the differences. But, if Sudanese-by-ancestry immigrant group participates in state, or neighborhoods' crime ad convicted of that crime: do they commit more crimes than that of Australian-born individuals or other ethnic minority group? Not really. Thus, we wanted to determine the probability that whether Sudanese-born individuals commit less or more crimes than their Australian-born individuals or of other ethnic minority groups in Victoria and Australia as a whole, supposedly, that the new piece of information is true.

For the purpose of this study, one reason as to why this study relies on Bayesian techniques is, it support for epistemic imports based on empirical evidence and probabilistic nature, following Ted Honderich (1995), works. Honderich believes that, past evidence, where further conclusions have to be drawn, merely remains as hypothesis; they are subject to further interpretations or investigations. And in other words, therefore, Oakes' conclusion has not. Thus, it lacks empirical evidence to rely on. I therefore take the press-on figures and Oakes' conclusion as hypothesis for this study; which I contend to do so through the Bayesian method for data analysis. I present a derivativation of the Bayesian analysis following; Bayes' rules, a manipulation of a conditional probability. A probability that one proposition is true Provided that, another proposition is true. For example, joint probability of two events, $\mathbf{A}$ and $\mathbf{B}$, can be expressed as: (see formula 1 \& 2 ): 


$$
\begin{aligned}
\mathrm{P}(A B) & =\mathrm{P}(A \mid B) \mathrm{P}(B) \\
& =\mathrm{P}(B \mid A) \mathrm{P}(A)
\end{aligned}
$$

In Bayesian probability, one of these 'events', is the hypothesis, $H$, and the other is data, $D$; and we wanted to test the relative truth of the hypothesis, given the data. We do this through the relations, in accordance with Bayes' rule, can be expressed as: (see formula 3):

$$
P(H \mid D)=\frac{P(D \mid H) P(H)}{P(D)}
$$

In this formula, $\mathrm{P}(H \mid D)$, represents the likelihood function, to assess the probability that the individual of Sudanese-born commit or is convicted of homicide crime or other relevant offences observed data arising from hypothesis. It expresses one's knowledge of how the likelihood of the Sudanese-born individuals committing a crime, or to be convicted of a crime would be; after taking into account the new piece of information, given that the hypothesis is true, is termed as, the posterior probability. For any proposition $\boldsymbol{S}$, $\mathrm{P}(S)$ will be used to represent "subjective probability", or our degree of beliefs. In particular, $\mathrm{P}(H)$ represents the estimate of the probability that Sudanese-born individuals committed a crime or to be convicted of, before taking-in new piece of evidence into account, is the prior probability; this is subjective aspect of the Bayesian probability theory. The term $\mathrm{P}(D)$, is obtained by summing or by multiplying $\mathrm{P}(D \mid H)$ by $\mathrm{P}(H)$, then divided by the value of $H$. This $\mathrm{P}(D)$ value, play roles of an ignorable, normalizing constant. For instance, there were 29,300 prisoners convicted of similar homicide offences in Australian-born. Of this, 77 out of 29,300 prisoners were of Sudanese-born backgrounds; 1,682 out of 29,300 prisoners were Australian-born individuals; and 27,541 out of 29,300 prisoners were of other ethnic minority groups in Australian community.

I should therefore, assume that the probability that Sudanese-born individual is convicted of homicide crime, is and it can be expressed as:

\section{Sudanese-Born Individuals}

$$
\begin{aligned}
\mathrm{P}\left(H_{1}\right) & =\mathrm{P}(\text { Sudanese-born individual is convicted of } \\
& \text { homicide crime }) \\
& =77 / 29,300 \\
& =\mathbf{0 . 0 0 2 6}
\end{aligned}
$$

\section{Australian-Born Individuals}

$$
\begin{aligned}
\mathrm{P}\left(H_{2}\right) & =\underset{\text { homicide offence })}{ } \\
& =1,682 / 29,300 \\
& =\mathbf{0 . 0 5 7 4}
\end{aligned}
$$

\section{Other Ethnic Minority Groups}

$$
\begin{aligned}
\mathrm{P}\left(H_{3}\right) & =\mathrm{P} \text { (an individual of other ethnic minority group } \\
& \text { is convicted of similar offences }) \\
& =27,541 / 29,300 \\
& =0.9400
\end{aligned}
$$

If $\boldsymbol{T}$, is the event that individuals convicted of homicide is of a Sudanese-born, or an Australian-born, or from-other ethnic group, then, it would be:

$\mathrm{T}=H_{1} \cup H_{2} \cup H_{3}$

And since these categories are mutually exclusive, the additive rule of probability may be applied:

$$
\begin{aligned}
\mathrm{P}(T) & =\mathrm{P}\left(H_{1} \cup H_{2} \cup H_{3}\right) \\
& =\mathrm{P}\left(E_{1}\right)+\mathrm{P}\left(H_{2}\right)+\mathrm{P}\left(H_{3}\right) \\
& =0.0026+0.0574+0.9400 \\
& =1.0000
\end{aligned}
$$

When probabilities of mutually 'exclusive events' sum to 1 , implies that the events are considered to be exhaustive. And thus, every individual in a prison survey populations fall into one of the three ethnic groups.

But now, suppose we tell you that the Australians' population aggregates, is $19,855,288$ persons. Let $\boldsymbol{D}$, represents the probability that an individual is convicted, given that he or she committed a crime, including homicide and other criminal offences, is and can be expressed as:

$$
\begin{aligned}
\mathrm{P}(D)= & 29,300 \text { (prisons' populations)/ 19,855, } 288 \\
& \text { (Australian populations) } \\
= & 0.0015
\end{aligned}
$$

Therefore;

$\mathrm{P}\left(H_{1} \mid D\right)=$ the probability that the Sudanese-born individual is convicted, given that he or she committed a crime, is: 
Table 1: Probability Estimates of Committing a Crime among Ethnic Groups

\begin{tabular}{|c|c|c|c|}
\hline Events & $\mathbf{P}(\mathbf{H})$ & $\mathbf{P}(\mathbf{D I H})$ & $\mathbf{P ( D I H ) P ( H )}$ \\
\hline \hline Sudanese-born $\left(\mathrm{H}_{1}\right)$ & 0.0026 & 0.000004 & $\mathbf{0 . 0 0 0 0 0 6 9 3}$ \\
\hline Australian-born $\left(\mathrm{H}_{2}\right)$ & 0.0574 & 0.0000847 & $\mathbf{0 . 0 0 3 2 4 1 1 9}$ \\
\hline Other ethnic minority $\left(\mathrm{H}_{3}\right)$ & 0.9400 & 0.0014 & $\mathbf{0 . 8 7 7 3 3 3 3 3}$ \\
\hline $\mathbf{P ( D )}$ & & & 0.0015 \\
\hline
\end{tabular}

$=77 / 19,855,288$

$=0.000004$

$\mathrm{P}\left(H_{2} \mid D\right)=$ the probability that an Australian-born individual is convicted, given that he or she committed similar offence, is:

$=1,682 / 19,855,288$

$=0.0000847$

And;

$\mathrm{P}\left(H_{3} \mid D\right)=$ the probability that the individual of other ethnic minority group is convicted, given that he or she committed similar offences, is:

$=27,541 / 19,855,288$

$=0.0014$

The probability of having being convicted of a homicide crime is lower (or 0.000004), among Sudanese-born individuals compared to that of Australian-born individuals and of other ethnic minority group. It is approximately; one-millionth, just, 1 in a million Sudanese-born person participates in a crime compared to that of Australian-born individuals, about: 0.0001 (1 in thousands), and 0.0014 ( 1 in hundreds) of other ethnic minority groups in Melbourne and Australia as a whole. This concludes that Sudanese-born individuals are 0.000004 times less or more likely to commit a crime, or to be convicted of homicide crime or other criminal offences than their Australian-born counterparts, and of other ethnic minority groups in Melbourne, Australia. $\mathrm{P}(D)$ :

Calculated probabilities are summed to obtained,

$$
\begin{aligned}
\mathrm{P}(D) & =\mathrm{P}\left(H_{1} \cap D\right)+\left(H_{2} \cap D\right)+\left(H_{3} \cap D\right) \\
& =\mathrm{P}\left(H_{1}\right) \mathrm{P}\left(D \mid H_{1}\right)+\left(H_{2)} \mathrm{P}\left(D \mid H_{2}\right)+\mathrm{P}\left(H_{3}\right) \mathrm{P}\left(D \mid H_{3}\right)\right. \\
& =0.000004+0.0014+0.0000847 \\
& =0.0014887, \text { or } \mathbf{0 . 0 0 1 5}
\end{aligned}
$$

\section{RESULTS}

The demographic characteristics are summarized in Table 1. The analytical sample includes three ethnic groups $\left(\mathrm{H}_{1} ; \mathrm{H}_{2}\right.$; and $\left.\mathrm{H}_{3}\right)$ in Australian prisons convicted of similar (same) offences. The probability that individual of Sudanese-born is convicted, given that he or she committed a crime: is 0.00000693 , or $0.000693 \%$. This is one-millionth; meaning, only one Sudanese-born in a million $(1: 1,000,000)$ is likely to commit or to be convicted of homicide crime in Australia compared to that of Australian-born individuals, about 0.00324119 , or $0.324119 \%$; and of other ethnic minority groups, about 0.87733333 or $87.33 \%$, which is about nine times more likely to commits or to be convicted of homicide crime or other criminal offences than their Sudanese-born and Australian-born counterparts.

Therefore, the probability that an individual of Sudanese-born is convicted, given that he or she committed a crime, is $\mathbf{0 . 0 0 0 0 0 6 9 3}$; check the working out below:

$$
\begin{aligned}
p(H \mid D) & =\frac{p\left(H_{1}\right) p\left(D \mid H_{1}\right)}{p(H 1) p(D \mid H 1)+p\left(H_{2}\right) p\left(D \mid H_{2}\right)+p\left(H_{3}\right) p\left(H_{3} \mid D\right)} \\
p\left(H_{1} \mid D\right) & =\frac{(0.000004 \times 0.0026)}{(0.000004 \times 0.0026)+(0.0574 \times 0.0001)+(0.9400 \times 0.001)} \\
& =0.00001
\end{aligned}
$$

$\mathbf{P}\left(\boldsymbol{H}_{1}\right)=$ the probability that individual of Sudaneseborn is convicted or imprisoned, given that he or she committed a crime

$=0.00000693(1: 100,000) ; \quad$ posterior probabilistic, Likelihood ratio

$\mathbf{P}\left(\boldsymbol{D} / \boldsymbol{H}_{1}\right)=$ the probability that an individual of Sudanese-born is convicted or imprisoned, given that he or she committed a crime

$=0.00001(1: 100,000) ;$ posterior probabilistic, Likelihood ratio 


\section{Likelihood Ratios (LRs)}

The LR of committing violent crime is the probability of that individual is convicted of homicide or other relevant criminals offence divided by the probability that individual is convicted, given that he or she committed the same offence; for example, $\mathrm{P}(D \mathrm{I} H) / \mathrm{P}(H)$, adjusted by $\mathrm{P}(D)$. A $\mathrm{P}(D)$ of 0.0015 , decreases the probability that individuals of the Sudanese-born commits or to be convicted of homicide or other relevant criminal offences by $99.99 \%$, and increases the probability that the Australian-born individuals commit a crime or to be convicted of the same offence by $99.7 \%$, and by $12.67 \%$ for other ethnic minority individuals. As such, the probabilistic change is continued to be influenced by a new piece of evidence-the knowledge, to find the "true estimated probability", that the individuals of anyspecific ethnic groups in Australian populations is convicted, given that he or she committed a crime or likelihood of high participation in states' or neighborhoods' crime rates. For example, the likelihood ratios: LRs of the Sudanese-born, are: 1:10,000 to 1: $1,000,000$; they are less likely to commit or to be convicted of homicide crime, insofar as, only 1 in a million or otherwise participates in a criminal activity compared to the likelihood ratios for Australian-born individuals, about 1:10,000 to 1:100; they are more likely to commit a crime or to be convicted of the same offences than their Sudanese-born counterparts. This concludes that an individual of Sudanese-born immigrant backgrounds in Australian community has a lower participation rates in the state or a neighborhoods' crimes than the Australian-born counterparts and other ethnic minority groups in Melbourne or in Australia as a whole.

\section{DISCUSSION}

This study is of the few studies to examine the probabilistic likelihood of the Sudanese-born immigrant youth committing a crime, or being convicted of homicide than those of Australians-born individuals or individuals of other ethnic minority groups in Victoria, Australia. The study first tested, the hypothesis that the rate of crimes and the likelihood of committing a crime or conviction of violence crime or homicide crime, would be significantly lower among Sudanese-born immigrant youth in Victoria and in Australia as a whole compared with that of the Australian-born individuals and of other ethnic minority counterparts. This hypothesis was confirmed with certain probability; the study found that compared with Australian-born individuals and that of other ethnic minority counterparts, as measured by the number of individuals convicted and recorded in Australian prisons' survey in 2009, individuals of Sudanese-born immigrant backgrounds were less likely to commit a crime or to be convicted of any relevant criminal offences, including homicide; only, 'one in a million' is likely or otherwise participates in a crime and to be convicted of the same offence in Melbourne, Australia than their Australian-born counterparts. This finding is consistent with the literature and emerging evidence, which suggests that Sudanese-born immigrant person is among the least participants in a states' or a neighborhood crimes, has a lower contribution to crime rates compared to Australian-born and of other ethnic minority counterparts (Collins 2005; Davidson 2011; Federation of Community Legal Centre, Flemington 2009). However, the effects of conviction and likelihood of committing homicide by Sudanese-born persons were mixed; the Victoria police data analysis revealed they are up-to 'eight' times more likely to commit a crime in Melbourne, and also, Dan Oakes' in an editorial report on crime rates in the state of Victoria, referred to the then Victoria police data, stated that Sudanese-born individuals they are up-to 'five' times more likely to be convicted or committing homicide crime in Melbourne, Australia than the Australian-born individuals and other ethnic minority counterparts. Although, a positive relationship between rates of crime perpetuated by Sudanese-born persons and their likelihood of committing or being convicted of homicide crime may be alarming and grossly irrational'surprising', it is inconsistent with other research (Nolan et al. 2009; AIC 2009), and may related to institutional biasness, racism, and perceived racial discrimination'anti-Sudanese' or 'anti-immigrant': resulting to overrepresentation of Sudanese immigrant populations in crime statistical data-being represented in numbers that are disproportionately higher than that of other groups. For example, one study found that host community ethno-racial discrimination and antiimmigrant behaviors and deleterious attitudes towards newly arrived ethnic minority group, might foster greater reliance on discriminatory and disproportionately representation in crime statistical data in state or neighborhood (Federation of Community Legal Centre 2009; Nolan et al. 2011; Thomas 2011).

The study then use content analysis of public discourse and the media representation of ethnospecific minority immigrant group in Australian community to test the hypothesis that increasing the 
intakes of refugees from Sub-Saharan African countries; Sudan, to be specific would significantly heighten the rates of crimes in Australians' states and territories compared to that if the intake is decreased or halt at all. Across all theoretical both: immigration and criminological theories and content analyses, as measured by Bayesian probabilistic theory or techniques, this hypothesis was not supported. The study found that the Australian-born-the native-born individuals had higher participation rates in crime both: in 'State' and 'nationwide' than that of Sudanese-born persons and of other ethnic minority-overseas-born counterparts in Melbourne, Australia. This finding is consistent with the literature and emerging evidence on immigration and criminality, which suggests that nativeborn individuals frequently engage in all ranges of criminal activities, committing more crimes than those of immigrant-overseas-born individuals in the community at entering and during the adjustment processes (Colic-Peisker \& Tilbury 2003; Berry 2001; Earnest 2008; Tonry 1994). However, the effects of the length of staying in a host country-or-community emerge to have mix effects on the rates of participation in crime or a crime perpetuated by an individual member of immigrant groups at different rates: the longer the immigrant persons stays in new community, the higher the participation rates in crime is likely be, but the shorter the stay, the lower the participation rates in state or neighborhoods' crime. Although a positive relationship between length of stays and the rates of participation in crime-deem perpetuated by immigrant individuals on native-born, or on member of general public it seems, it is inconsistent with other empirical research and theoretical analysis of 'immigration and delinquency'-assessing whether immigrant commits more crime than native-born and vice versa. For example, one class theoretical study on immigration and crime found that native-born individuals had a higher participation rates in crime than that of overseas-born or that of immigrant group member (Chen \& Zhong 2013; Collins 2005; Ignacio et al. 2010); irrespective of the length of stays in their new hosting community-Australia, in this case.

The focus on Sudanese immigrant in Australians' populations, and in particular, who is identified as 'Sudanese-born' and 'Sudanese-by ancestry' (ABS 2006: 2010), is based on the premises that such Characteristic of identification is ambiguous, in three ways: (1) Sudan is a home to Ethiopian, Eritrean and Somalis refugees; (2) Refugees have their first generation of Sudanese-born; (3) 'Sudanese-born refugees' have another generation of 'Sudanese-born' of their own. For example, the Australian Bureaus of Statistic (ABS) recorded Sudanese immigrant community in two categories: the Sudanese-by-birth and the Sudanese-by ancestry (ABS 2006:2010); perhaps misleading, is associated with pervasive crime rates and the likelihood of Sudanese Immigrant persons committing a crime or convicted than their Australian-born counterparts. This, however, is disputable. The community is distinctively, 'Sudaneseby-ancestry'. The country, Sudan hosted refugees and immigrants from Ethiopia, Eritrea and the Somalia, five decades ago before resettling to Australia; and whose young members of the respective communities are classified as: Sudanese-born. And hence the differences, their participations in criminal activities or other antisocial behaviors that deemed socially unacceptable in Australian society are Accounted for the 'Sudanese-by-ancestry' immigrant group or community contribution into a states,' or neighborhoods' crimes; and be identified to have a higher and substantial participants in criminal events in Victoria or Australia as a whole. While available Evidence suggested that, the police department and other statistical data management authorities such as the Australian Bureau of statistic, recording data is based on the country of birth as meaningful indicator of criminal activity in the community or the state (ABS 2010; Australian Institute of Criminology, AIC 2010). Thus, who is identified as 'Sudanese-born' in Australian data management bodies, is unclear, however, controversial one, whether it is or not: an 'EthiopianSudanese-born', or the 'Eritrean-Sudanese-born', or the 'Somalis-Sudanese-born'; or 'Sudanese-Sudaneseborn' in Australian populations, is poorly understood, given that there is no sufficient evidence or existing literature to ensure the conclusion on, which group is identified as 'Sudanese-born'; or perhaps, a constitutive of those groups. Even though, this finding and emerging evidence have confirmed the hypothesis that Sudanese-born individuals are less likely to commit a crime or convicted of homicide than Australian-born individuals and other ethnic minority counterparts, more research is needed to investigate the question of: 'who is identified as Sudanese-born?' in Australian population. Hence, it would have been preferable to have other measure of this variable that was independent of law enforcement records, for example, neighborhoods surveys to helps identify and redefines a community group-as: the Sudanese-born and Sudanese-ancestry. 


\section{LIMITATIONS AND FUTURE RESEARCH DIRECTIONS}

The immigrant data lacks some significant variables. Firstly, the prison data, for instance, lacks records on: age, gender, educational levels, marital status, skilled or unskilled labor, prior to imprisonment in Australian's prison populations were entirely missing; and secondly, the ABS data carries two sub-categories of the Sudanese immigrant: the Sudanese-by-ancestry and Sudanese-by-birth (SB). However, the analysis on the SB sub-category makes the sample size small, with fewer variables, and therefore, it cannot be accurately generalized. That is to say, however, the variable ' $S B$ ' is 'ambiguous' one: poorly understood and un-identified variable. And although, other ethnic minority groups in Australian prisons' survey has included; an Eritrean, the Ethiopians and the Somalis, who before arriving to Australia were refugees in Sudan for a significant periods of time. They, therefore, have Sudanese-born (SB) of their own (first-generation) in, their respective communities; yet, they are not necessarily a 'Sudanese', in the sense of media representation of Sudanese immigrant community in Australia and also, a recording and presentation in crime statistical data both: the state and national level. This should be considered in future studies on Sudanese immigrant populations in Australia. Finally, the paper bases its objectives primarily on one state's population (Victoria's'), only out of five (5) administrative states and two (2) territories, which makes Australia, and thereby, cutting a significant population of the Sudanese immigrants countrywide. Hence, further and more research is sorely needed for better understanding of this immigrant group and how they adjust to their new adopted Australian community.

\section{CONCLUSION AND RECOMMENDATIONS}

In the findings, the following conclusions are made, despite Australia being a liberal society and presumably without ethnic criminals, Sudanese immigrants youth are continue to be regarded as gangs; and therefore, there are ethnic criminals in Australia. However, that might be the position, appears insupportable. As such, the idea seems to be not an attitude directed towards the immigration itself; but rather, an attitude directed towards race or ethnicity, which might mean that, the Sudanese-born immigrant youth are by nature or by appearance, a criminal, but, rather not on the basis of the crimes committed by individuals in this group.

Despite the media reportages of the Sudanese immigrant youth heightening the crime rates in Victoria; an impact supported by no empirical evidence, rather engaging in a primitive accumulation of negative perceptions about ethno-specific group-just on basis for pointing a finger to them, blaming them for much wrong even if they did not take part-just only that they are new and they look different-visible immigrant groups. The Australian statistical agencies (ABS), Victorian Police recorded incidences and the prison data gathered for this analysis reveals a significant decline, rather than the increases for years of 2006 and 2007 crime rates. Neither do theoretical imports or existing literature reveal race, as an issue in committing crimes and rather, it is a behavior provided or adopted from the hosting society-Australian community, where this immigrant group resides. Therefore, there is no significant correlation between 'ethnicity' and criminality', as both Fraser and Oakes contend on the Sudanese immigrants in Australia. That is to say, however, there is no doubt, that crimes do affect all people significantly. And as shown, the immigrant community, however, misrepresented, marginalized from the mainstream Australians' populations and somewhat deliberately excluded by the media portrayal-analysis on the existing data, does reveal a gross lack of empirical evidence, that is supportive to either Oakes' or Frasers' claim. As such, the youth lack proper access to the media or other social settings of the society, fearing insults or outright suspicion from the members of the public. And therefore, attempts to provide accurate, empirically predictive generalization should aim at providing the empirical evidence, supportive of either or both claims. Moral panic exacerbates the immigrant acculturative stress, as they are more predisposed to psychological imports of hates-and could significantly make their integration efforts difficult. In the same way, intervention(s) or other ameliorating mechanisms, accommodative to the immigrants should culturally be relevant to the targeted immigrant group. Finally, the recorded data record is based on the country of birth, as meaningful; perhaps, a meaningless and insignificant indicator variable of criminal activity in the community or a residing state. It would have been preferable to have multiple quantitative variables, which are bias free, to achieve accuracy and reliability in data analysis, and free from imperialistic tendency of singling-out ethno-specific group by overrepresentation or disproportionately presentation of immigrant group, including, Sudanese immigrant populations in Australian community, but rather an independent law enforcement recording data set or volume management. For example, neighborhood surveys, would have positive impacts, have better understanding of immigrant- 'ethnic' minority group and how their children (children of 
immigrant) adjust to newly adopted countrycommunity; and to understands what this group constitutes of; group dynamic and their culturesgoverning them; understanding their views about social setting, social and intercultural interactions, would definitely have significant effects on identifying 'what' and 'who', and then redefines, this community group entirely-as either or both: the 'Sudanese-by-birth' and 'Sudanese-by-ancestry' respectively.

\section{REFERENCES}

Ager, A., \& Strang, A. 2008. 'Understanding Integration': A conceptual framework, Journal of Refugee Studies, Vol.21, No.2, pp.166-191. http://dx.doi.org/10.1093/jrs/fen016

Australian Bureau of Statistic of Crimes [cited as ABS, 2006-2007; 2010-2012], accessed on august the $2^{\text {nd }} 2013$ from: http: www.ABS.gov.au

Australian Institute of Criminology (AIC 2007-009 Report), "Crimes: Figures and Facts". Accessed on 19th July 2014 from; www.aic.edu.au

Berkman, L. F., \& Kawachi, I. 2000. Social integration, social networks, social support and health: Social Epidemiology, New York: Oxford University Press, pp. 137-173.

CMYI. 2006. Refugee Young People and Resettlement. Melbourne: Centre for Multicultural Youth Issues.

Camarota, S, A and Vaughan, J, M 2009, Immigration and Crime Assessing a Conflicted Issue, accessed on $12^{\text {th }}$ August 2014 from: http://www.cis.org/articles/2009/crime.pdf

Chen, $X$ and Zhong, H. 2013. 'Delinquency and Crime among Immigrant Youth': An Integrative Review of Theoretical Explanations, Vol. 2, pp. 210-232

Collins, J. 2005.Ethnic Minorities and Crime in Australia: Moral Panic or Meaningful Policy Responses: Retrieved on $2^{\text {nd }}$ August 2014.

Cook, D, 4th October 2007. Sudanese no more prone to crime than any other group in the community; Accessed on 23rd July, 2014 from: http://web.ebscohost.com/ehost/detail?vid=13\& sid=2aef5

Davidson, S. 2011. Refugee crime wave nothing but hogwash: accessed on $10^{\text {th }}$ September 2014;from: www.theage.com.au

DIAC. 2009. Settlement Database. Department of Immigration and Citizenship, ACT; accessed on $10^{\text {th }}$ November 2014, from: www.diac.gov.au

Federation of Community Legal Centres, 2009. "Flemington \& Kensington Community Legal Centre - Race Discrimination Case Documents - Haile-Michael v Konstantinidis" Retrieved $2^{\text {nd }}$ August 2014

Fraser, A.2005.Professor Fraser fights for free speech: Ironbark resources; accessed on $16^{\text {th }}$ August 2014,from: www.ironbarkresources.com

Gifford, S., Bakopanos, C., Kaplan, I., \& Correa-Velez, I. 2007. 'Meaning or measurement?' Researching the social contexts of health and settlement among newly arrived refugee youth in Melbourne, Australia. Journal of Refugee Studies, Vol.20, No.3, pp.414-440.

http://dx.doi.org/10.1093/jrs/fem004

Gifford, S., Correa-Velez, I., \& Sampson, R. 2009. 'Good Starts for recently arrived youth with refugee backgrounds': Promoting wellbeing in the first three years of settlement in Melbourne, Australia. Melbourne: La Trobe Refugee Research Centre.

Grace K, and Marta 1995. 'Optimism and Achievement': The Educational Performance of Immigrant Youth. Social Science Quarterly 76: 1995, pp. 1-19.

Honderich, T. 1995. The Oxford Companion to Philosophy: Oxford University Press.

Krieger, N., Smith, K., Naishadham, C., \& Barbeau, E. M. 2005. 'Experiences of Discrimination': Validity and reliability of a self-report measure of population health research on racism and health. Social Science and Medicine, Vol. 61, pp.15761596.

http://dx.doi.org/10.1016/j.socscimed.2005.03.006

Mesch, G. S., Turjeman, H., \& Fishman, G. 2008. Perceived discrimination and the wellbeing of immigrant adolescents. Journal of Youth and Adolescence, Vol. 37, No.5, pp. 592604.

http://dx.doi.org/10.1007/s10964-007-9210-6

Montgomery, E., \& Foldspang, A. 2008. Discrimination, mental problems, and social adaptation in young refugees. European Journal of Public Health, Vol.18, pp. 156-161. http://dx.doi.org/10.1093/eurpub/ckm073

Myers, J., L., Well, A., D., \& Loch, jr, R., F. 2010. Research Design and Statistical Analysis, $3^{\text {rd }}$ edn, Harvard University Press.

Nolan, D., Farquharson, K., Marjoribanks, T., \&Muller, D. 2011. 'Mediated multiculturalism': Newspaper Representation of Sudanese migrant in Australia, Journal of Intercultural studies, Vol.32, No.2, pp.655-671.

O'Sullivan, K., \& Olliff, L. 2006. 'Settling in': Exploring good settlement for refugee young people in Australia. Melbourne: Centre for Multicultural Youth Issues.

Olliff, L., \& Couch, J. 2005. 'Pathways and Pitfalls': The Journey of Refugee Young People in and Around the Education System in Greater Dandenong, Victoria. Youth Studies Australia, Vol. 24, No.3, pp. $42-46$.

Oakes, D $20^{\text {th }}$ August 2012. 'African youth crime concern', Accessed on July the 20th, 2014 from: http://www.theage.com.au/ victoria/African-youth

Oakes, D, 20th August 2012. 'Fear of Cronulla-like unrest as refugee lawlessness grows in Melbourne': the age newspaper, retrieved on $24^{\text {th }}$ July 2014 from: http.

Sinclair, D 2009. "Australia arrests 'foil army base terror plot": the Independent, retrieved on 2nd August 2013 from: http://www.theage.com.au/victoria

Sellin, T 1938. 'Culture Conflict and Crime': Social Science Research Council: New York, USA.

Thomas, M. 2011. 'Theoretical Articulation on Immigration and Crime': Homicide Studies Vol.15, pp.382-403. http://dx.doi.org/10.1177/1088767911424539

Tran, N. 2005. 'Immigration and Crime': Do Asian Immigrants Bring More Crimes to Australian Society? School of Public policy, Australian National University, ACT, Australia.

\section{DOI: http://dx.doi.org/10.6000/1929-4409.2015.04.09}

(C) 2015 Atemthii D. Dau; Licensee Lifescience Global.

This is an open access article licensed under the terms of the Creative Commons Attribution Non-Commercial License (http://creativecommons.org/licenses/by-nc/3.0/) which permits unrestricted, non-commercial use, distribution and reproduction in any medium, provided the work is properly cited. 